14,11

\title{
Тепловой эффект перехода моноклинной фазы в орторомбическую в сверхвысокомолекулярном полиэтилене
}

\author{
(С) В.М. Егоров ${ }^{1}$, В.А. Марихин ${ }^{1}$, Л.П. Мясникова ${ }^{1}$, А.К. Борисов ${ }^{1}$, Е.М. Иванькова ${ }^{2}$, С.С. Иванчев ${ }^{2}$ \\ ${ }^{1}$ Физико-технический институт им. А.Ф. Иофрфе, \\ Санкт-Петербург, Россия \\ ${ }^{2}$ Институт высокомолекулярных соединений РАН, \\ Санкт-Петербург, Россия \\ E-mail: victor_egorov1@inbox.ru
}

Поступила в Редакцию 30 мая 2019 г.

В окончательной редакции 30 мая 2019 г.

Принята к публикации 4 июня 2019 г.

Проведен анализ тепловых эффектов, сопровождающих твердотельный фазовый переход в сверхвысокомолекулярном полиэтилене из нестабильной моноклинной в термодинамически устойчивую орторомбическую фазу. Предложенная модель строения надмолекулярных образований моноклинной фазы не противоречит рентгенографическим данным, полученным ранее на этом полимере.

Ключевые слова: сверхвысокомолекулярный полиэтилен, калориметрия, фазовый переход, моноклинная фаза.

DOI: $10.21883 /$ FTT.2019.10.48278.500

Промышленное производство сверхпрочных волокон на основе сверхвысокомолекулярного полиэтилена (СВМПЭ), являющихся рекордсменами по удельной прочности, в настоящее время базируется на использовании метода гель-технологии, затратном и экологически вредном. Рассматриваются возможности получения сверхпрочных волокон на основе СВМПЭ более дешевым и экологически безопасным способом. Наиболее перспективным методом для решения этой задачи считается производство волокон непосредственно из порошкообразных продуктов синтеза - реакторных порошков (РП), путем их монолитизации (компактировании) и последующей ориентационной вытяжки [1-4]. В предлагаемом методе наиболее дискуссионным является вопрос о параметрах надмолекулярной структуры, контролирующих способность полимера к достижению высоких ориентационных удлинений, поскольку оказалось, что не из всех РП получаются высокопрочные высокомодульные нити. В частности, одним из критериев пригодности РП к получению из них сверхвысокопрочных высокомодульных нитей рассматривается присутствие в РП структурно стабилизированной кристаллической моноклинной фазы, свидетельствующей, как полагают [5], о специфическом строении неупорядоченных областей, обогащенных распрямленными проходными сегментами молекулярных цепей. В таких РП меньше молекулярных зацеплений, отрицательно сказывающихся на деформационно-механических свойствах реакторного порошка $[6,7]$.

Моноклинная фаза в полиэтилене является термодинамически нестабильной и превращается в стабильную орторомбическую фазу (ОФ) в зависимости от времени и температуры. Это указывает на то, что фазовый переход первого рода контролируется механизмом, имеющим релаксационный характер. В работе [5] высказано предположение, что МФ в полимере удерживается напряжениями, которые передаются кристаллитам через натянутые проходные молекулы, соединяющие соседние кристаллиты в ламелях или фибриллах. Существующее в полиэтилене даже при комнатной температуре сегментальное движение приводит к релаксации напряжения и переходу МФ в ОФ. Произойти это может при комнатной температуре в течение нескольких недель [6]. При более высоких температурах этот процесс наблюдается значительно быстрее [5].

Целью настоящей работы является исследование тепловых эффектов при фазовом переходе МФ $\rightarrow$ ОФ. Объектом исследования методом дифференциальной сканирующей калориметрии (ДСК) служили таблетки из РП молекулярной массы $6.5 \cdot 10^{6} \mathrm{~g} / \mathrm{mole}$, компактированные при комнатной температуре в течение $15 \mathrm{~min}$ под давлением $15 \mathrm{MPa}$ [7]. Тепловые измерения выполнили на калориметре ДСК-500 (ООО „Спецприбор“, Саратов), прокалиброванном по температурам плавления индия $(429.8 \mathrm{~K})$ и льда $(273.1 \mathrm{~K})$ и теплоемкости сапфира.

На рис. 1 представлена кривая ДСК, полученная при нагревании образца из РП весом $2.5 \mathrm{mg}$. На кривой зафиксирован тепловой эффект, отвечающий процессу плавления - эндотермический пик с температурой максимума $T_{\max }$ в сравнительно узком температурном интервале $\Delta T=T_{2}-T_{1}$, где $T_{1}-$ температура начала процесса плавления, получаемая по точке пересечения линейной экстраполяции переднего фронта пика с базовой линией; $T_{2}$ - температура окончания процесса плавления. Известно, что температурные параметры пиков на кривой ДСК завышены вследствие термического 


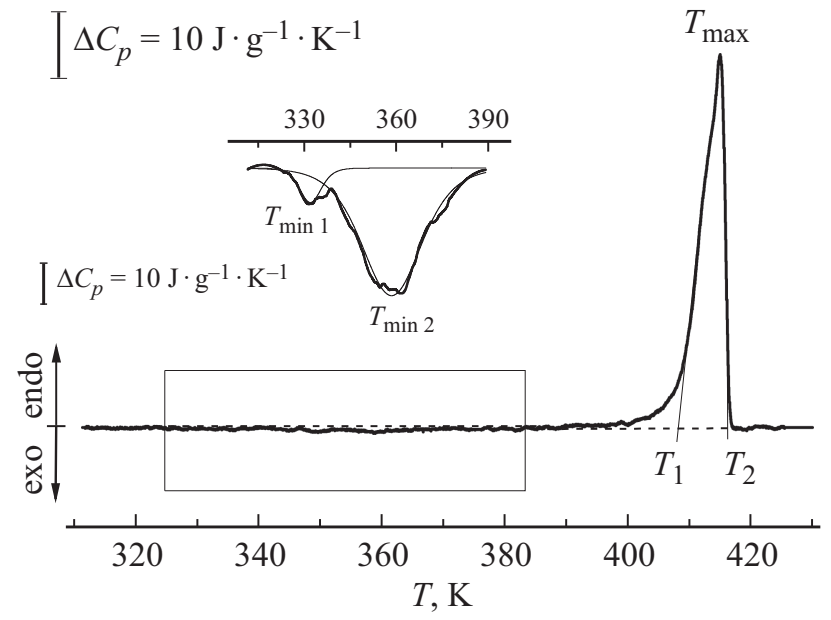

Рис. 1. Кривая ДСК образца таблетки из РП весом $2.5 \mathrm{mg}$. Скорость нагревания $V=2 \mathrm{~K} / \mathrm{min}$. На вставке - часть кривой ДСК, расположенная внутри обозначенного на рисунке прямоугольника. Жирная линия - экспериментальная кривая, тонкие линии - расчетные.

запаздывания. Устранение этого термического запаздывания по методике $[8,9]$ позволило определить истинные значения $T_{1}=409.0 \mathrm{~K}, T_{\max }=412.5 \mathrm{~K}, T_{2}=413.5 \mathrm{~K}$ и $\Delta T=4.5 \mathrm{~K}$.

Помимо температур по кривой ДСК определена энтальпия плавления образца $\Delta H_{m}=225 \mathrm{~J} / \mathrm{g}$ (по площади эндотермического пика в интервале температур $T_{1}-T_{2}$ ) и степень кристалличности $\chi=\Delta H_{m} / \Delta H^{0}=77 \%$, где $\Delta H^{0}=292 \mathrm{~J} / \mathrm{g}$ - энтальпия плавления совершенного кристалла $[10]$. Эндотермический тепловой эффект, проявляющийся в виде примыкающему к пику плавления „Плеча“ со стороны низких температур (ниже температуры $T_{1}$ ) связан с размораживанием сегментального движения в неупорядоченной фазе полимера [8]. Поэтому в расчет энтальпии плавления и степени кристалличности он не включался.

Пик плавления на кривой ДСК использовали для оценки еще одной характеристики надмолекулярной структуры полимеров - параметра внутрицепной кооперативности плавления (v), физический смысл которого состоит в том, что он определяет минимальную последовательность из $v$ повторяющихся звеньев в цепи, переходящих как целое из кристаллита в свернутое состояние статистического клубка в расплаве $[11,12]$. Оценку $v$ производили по формуле $v=2 R\left(T_{\max }\right)^{2} / \Delta T \Delta H^{0}$, где $R$ - газовая постоянная. Безразмерный параметр $v$ выражается числом $\mathrm{CH}_{2}$ - групп в транс-участке цепи ПЭ, одновременно участвующих в акте плавления. Если умножить $v$ на длину единичной связи $\mathrm{C}-\mathrm{C}$ (размер проекции $\mathrm{C}-\mathrm{C}$ связи основной цепи на ось макромолекулы $h=0.124 \mathrm{~nm}$ ), то параметр $L_{0}=v \times h$ можно сопоставлять с параметрами надмолекулярной структуры, например, с размерами ламелярных образований [9]. Для испытываемого образца величина $L_{0}$, определенная по полученному значению параметра кооперативности, равна $19 \mathrm{~nm}$. Эта величина сопоставима с толщиной ламелярных кристаллов полиэтилена, получаемой по соотношению Томсона-Гиббса для температуры плавления $T_{1}$, то есть $\sim 18-20 \mathrm{~nm}$.

В работе [5] на рентгеновском дифрактометре исследовались как таблетки из того же РП, что и в настоящей работе, так и в отдельной, достаточно крупной частице исходного РП, не подвергнутой предварительной механической обработке. Оказалось, что в обоих случаях в испытываемых образцах присутствует МФ. Содержание МФ в частице исходного РП оказалось незначительно (около $2 \%$ от кристаллической составляющей, принятой за 100\%). При нагреве оно постепенно уменьшалось и при температуре $\sim 340 \mathrm{~K}$ моноклинная фаза полностью переходила в орторомбическую. В таблетке компактированного РП моноклинная фаза оказалась более стабильна и ее содержание составляло $\sim 24 \%$. Для фазового перехода МФ $\rightarrow$ ОФ в этом случае были необходимы более высокие температуры отжига, вплоть до температуры $370 \mathrm{~K}$. Проанализировав полученные данные, авторы пришли к выводу, что причина возникновения МФ в обоих случаях различна: МФ в исходном РП связана с наличием проходных натянутых молекул между кристаллами, образовавшихся при кристаллизации в условиях ограниченного пространства; в компактированных таблетках появление МФ обусловлено механическим воздействием при компактировании.

Поскольку энергия перехода ОФ $\leftrightarrow$ М, рассчитанная теоретически, достаточно мала [13], и, как показано в работе [5], этот фазовый переход в СВМПЭ происходит в широком температурном диапазоне, на кривой ДСК в этом температурном диапазоне следует ожидать незначительный по отклонению от базовой линии экзотермический эффект. Действительно, при многократном увеличении участка кривой ДСК, обозначенного на рис. 1 прямоугольной областью, этот эффект обнаруживается (см. вкладку на рис. 1). Можно предположить, что этот эффект связан с фазовым переходом МФ $\rightarrow$ ОФ, поскольку он имеет, в отличие от плавления, экзотермический знак, характерный для перехода термодинамически нестабильной фазы в стабильную. Дополнительные аргументы в пользу предположения о наблюдаемом переходе, как о переходе первого рода будут приведены ниже. Из рисунка видно, что экзо-эффект проявляется в виде двух пиков.

Анализ пиков, полученных вышеуказанным способом, проводился в рамках термодинамической теории самосогласованного поля [14-16] применительно к $\Lambda$-образным размытым ФП первого рода. Теория анализирует образование и последующий рост устойчивых зародышей новой фазы в матрице старой фазы. В объеме старой фазы происходит локализация многочисленных флуктуаций в ограниченном объеме в виде стабильных зародышей новой фазы, так называемых элементарных объемов перехода $\omega$. Размеры устойчивых зародышей можно 
Термодинамические параметры фазового перехода МФ $\rightarrow$ ОФ

\begin{tabular}{c|c|c|c|c|c}
\hline $\begin{array}{c}\text { Пик } \\
\text { в дублете }\end{array}$ & $\begin{array}{c}T_{0}, \\
\mathrm{~K}\end{array}$ & $B$ & $\begin{array}{c}\Delta C_{\max }, \\
\mathrm{J} / \mathrm{gK}\end{array}$ & $\begin{array}{c}q_{01}, q_{02}, \\
\mathrm{~J} / \mathrm{g}\end{array}$ & $\begin{array}{c}\omega_{1}, \omega_{2}, \\
\mathrm{~nm}^{3}\end{array}$ \\
\hline 1 & 332.0 & 140 & 0.13 & 1.2 & 53.0 \\
2 & 358.5 & 55 & 0.48 & 12.4 & 2.2
\end{tabular}

определить из формы пиков $C_{p}$, соответствующих переходам первого рода. В работе [17] получено соотношение для температурной зависимости теплоемкости при размытом фазовом переходе в виде

$$
\begin{aligned}
\Delta C_{p}(T)= & 4 \Delta C_{m} \times \exp \left[B\left(T-T_{0}\right) / T_{0}\right] \\
& \times\left[1+\exp \left[B\left(T-T_{0}\right) / T_{0}\right]\right]^{-2},
\end{aligned}
$$

где $T_{0}$ - температура ФП первого рода, $\Delta C_{m}-$ максимальное значение теплоемкости при $T=T_{0}, B-$ атермический параметр. Параметр $B$ в приведенном выше соотношении связан с величиной пика теплоемкости $\Delta C_{m}=q_{0} \times B / 4 T_{0}$ и элементарным объемом превращения $B=\omega \rho q_{0} / k T_{0}$, где $q_{0}$ - теплота превращения, $k-$ постоянная Больцмана, $\rho-$ плотность. Из приведенных соотношений можно найти $\omega$ (полагая в первом приближении, что плотность кристаллов полиэтилена $\left.\rho \sim 0.9 \mathrm{~g} \cdot \mathrm{cm}^{-3}[10]\right)$. Таким образом параметр $B$ оказывается структурно чувствительным параметром, поскольку определяет в материалах с размытыми ФП объемы зародышей новой фазы.

Разделение дублета, изображенного на вкладке рис. 1 на два пика симметричной $\Lambda$-образной формы, то есть зависимости $q_{1}(T)+q_{2}(T)$, где $q_{01}=\int q_{1}(T) d T$ и $q_{02}=\int q_{2}(T) d T$, проводилось при условии равенства энтальпии $\Delta H$ экспериментально полученного пика сумме энтальпий $\left(q_{01}+q_{02}\right)$ двух симметричных пиков. Наилучшее совпадение расчетных и экспериментальных зависимостей наблюдалось при значениях параметров, указанных в таблице. Результаты расчета $\Lambda$-образных пиков по соотношению (1) показаны на этом рисунке в виде тонких линий.

На рис. 2 совмещены данные, полученные в настоящей работе в виде зависимости $q_{1}(T)+q_{2}(T)$, и привлеченные из литературы зависимости содержания МФ от температуры отжига в компактированном РП $(a)$ и в частице исходного РП $(b)$ [5]. Из рисунка видно, что точки достаточно хорошо совмещаются с кривыми, что позволяет сделать очевидные выводы. Во-первых, это подтверждает сделанное предположение о том, что наблюдаемый на кривой ДСК экзотермический эффект обусловлен фазовым переходом, и, во-вторых, позволяет сопоставить величину полученного эффекта с энергией фазового перехода $М \Phi \rightarrow$ ОФ. Действительно, если использовать степень кристалличности $\chi$ моноклинной фазы, экспериментально определенную рентгенографическим методом [6] и равную 24\% (см. рис. 2,a), то $100 \%$ этой фазы составят величину $\left(q_{01}+q_{02}\right) / 0.24 \approx 0.25 \mathrm{kcal} / \mathrm{mole}$, сопоставимую по порядку величины с литературными данными [13].

При рассмотрении возможных локальных мест возникновения устойчивых зародышей новой фазы необходимо учитывать их форму и местоположение в различных надмолекулярных образованиях СВМПЭ. Как видно из рис. 3 , надмолекулярная структура СВМПЭ состоит в основном из двух элементов - ламелей и фибриллярных образований. По мнению авторов рабо-
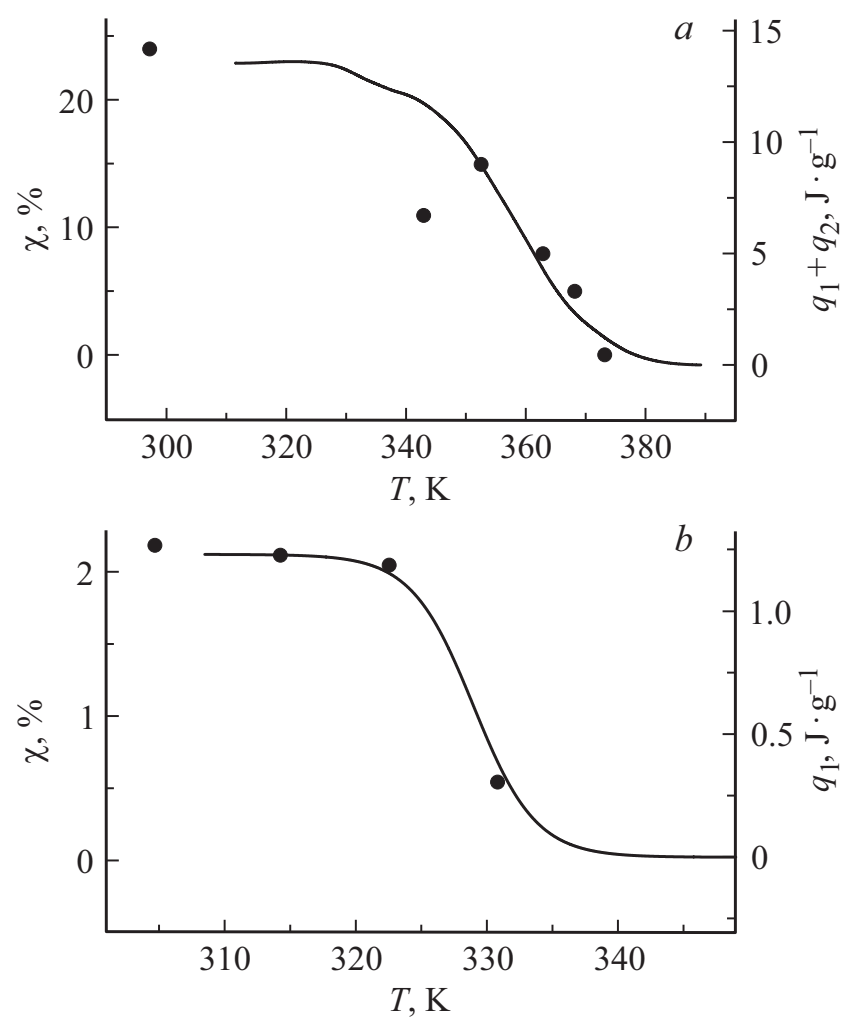

Рис. 2. Зависимости содержания МФ от температуры отжига в компактированном РП $(a)$ и в частице исходного РП $(b)-$ точки [7]; зависимости избыточной энтальпии $\left(q_{1}+q_{2}\right)(a)$ и $q_{1}(b)$ от температуры - линии.

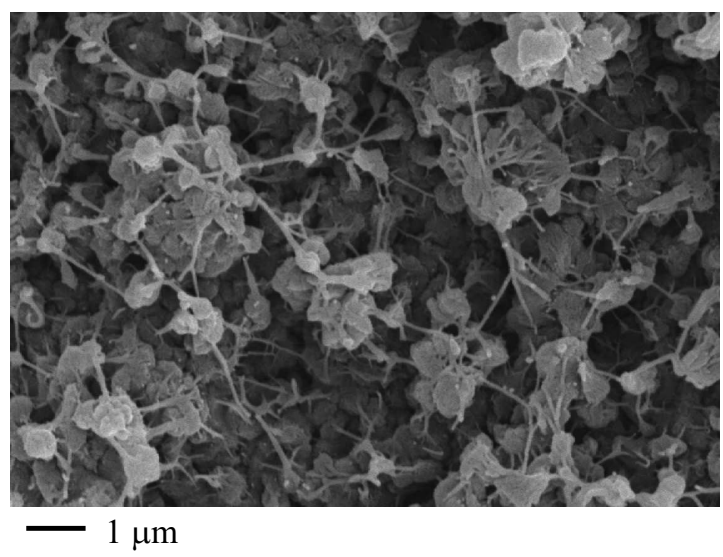

Рис. 3. Электронно-микроскопический снимок таблетки из РП. 
ты [5] МФ в исходном РП связана с наличием проходных натянутых молекул между кристаллами в ламелях или в фибриллах, образовавшимися при кристаллизации в условиях ограниченного пространства. По-видимому, эти проходные молекулы должны быть не одиночными, а находиться в составе некоего „пучка“, способного давать когерентное рассеяние в рентгеновской дифракции. Эти проходные „пучки“ или „связки“ молекул могут иметь существенно меньшие поперечные размеры, чем видимые на электронных фотографиях макрофибриллы. Такие образования можно условно назвать наномостиками. Исходя из этой позиции, наблюдаемый фазовый переход как в макрофибриллах, так и в наномостиках связан с тепловым эффектом на первой стадии фазового превращения, то есть с элементарными объемами превращениями $\omega_{1}=53 \mathrm{~nm}^{3}$.

Природа появления МФ в компактированных таблетках по мнению авторов работы [5] связана с механическим воздействием и пластической деформацией, которая не затрагивает фибриллы и кристаллические сердечники ламелей. Пластическая деформация происходит за счет межламеллярных прослоек. Различные элементы этих прослоек - слабо- и сильноизогнутые петли, проходные натянутые и не натянутые молекулы деформируются и передают механическое напряжение кристаллическим сердечникам ламелей, в результате чего появляется нестабильная МФ. При снятии внешней нагрузки в этих элементах остаются остаточные напряжения. Эти остаточные напряжения создаются энтропийными силами и с течением времени и при повышенной температуре могут релаксировать. Релаксация этих напряжений вызывает обратный переход неравновесной МФ в ОФ. Исходя из этого, можно предположить, что этот обратный переход связан с тепловым эффектом на второй стадии фазового превращения с элементарными объемами превращениями $\omega_{2}=2.2 \mathrm{~nm}^{3}$ внутри кристаллических сердечников лемелей.

Как отмечалось выше, необходимо учитывать не только местоположение зародышей новой фазы в надмолекулярной структуре полимера, но и форму или габитус этих зародышей. При полимеризации в условиях ограниченного пространства в фибриллярных образованиях МФ препятствует образованию молекулярных зацеплений [6,7], поэтому объем зародыша МФ не содержит этих зацеплений. Боковая часть фазовой границы также, как и в ламелях, проходит через относительно слабые вандерваальсовы связи между метиленовыми группами соседних молекул. Другая часть фазовой границы, необходимая для образования объема новой фазы, проходит по торцевой поверхности параллельно упакованных молекул. В этой торцевой поверхности, по-видимому, концентрируются молекулярные зацепления, для преодоления которых при вытяжке в предельном случае необходим разрыв связи $\mathrm{C}-\mathrm{C}$.

В работе [18] показана общность термодинамических свойств нормальных длинноцепочечных алифатических соединений и полиэтилена. Эта общность состоит в том, форма или габитус зародыша фазового превращения определяется соотношением энергий когезии на боковой и торцевой поверхности элементарного зародыша. Продольный размер зародыша $L$ с прямоугольным основанием $b$, где $L=\omega \times b^{-2}$, определяется соотношением [19]:

$$
L=\omega^{1 / 3} \times\left(E_{k n} / E_{\kappa 1}\right)^{2 / 3},
$$

где $E_{\kappa 1}$ и $E_{k n}-$ энергии когезии метиленовой группы $\mathrm{CH}_{2}$ и концевых групп, соответственно. Для фибриллярных образований $E_{\kappa 1}$ соответствует энергии когезии, приведенной в монографии [20] для вкладов от $\mathrm{CH}_{2}$-групп и равной $3.6 \mathrm{~kJ} / \mathrm{mol}$. В качестве концевой группы в фибриллярных образованиях можно считать узел молекулярного зацепления. В предельном случае, когда этот узел нельзя распустить без разрыва полимерной молекулы, за энергию когезии можно принять энергию разрыва $\mathrm{C}-\mathrm{C}$-связи, равную $348 \mathrm{~kJ} / \mathrm{mol}$. Расчет по соотношению (2) дает для величины $L$ значение $\sim 80 \mathrm{~nm}$. Зародыши таких размеров могут находиться как в макрофибриллах, имеющих продольные размеры несколько сотен nm и видимые в растровом электронном микроскопе (см. рис. 3), так и в наномостиках, соединяющих несколько соседних ламелей и не видимых на вышеприведенных микрофотографиях. Исходя из полученного предельного значения $L$ эти наномостики должны проходить через стопку из $\sim 4$ одиночных ламелей размером $L_{0}$.

Рассмотрим не предельный случай, когда в образовании торцевой поверхности зародыша новой фазы участвуют другие молекулярные зацепления, преодоление которых не требует разрыва молекулярной цепи. Движение основной цепи полимера происходит по поворотно-изомерному механизму, когда при вращении происходят перескоки из одной потенциальной ямы в другую. В молекулярных зацеплениях участвуют как минимум две полимерные цепи, каждая из которых имеет в точке зацепления набор дефектов в виде чередующихся в разной последовательности гош- и транс-изомеров, отвечающих положению в одной из потенциальных ям. При этом транс-изомеры $(t)$ обладают минимальной энергией, а два гош-изомера $\left(g\right.$ и $\left.g^{*}\right)$ различаются направлением углов поворота, но обладают одинаковой повышенной потенциальной энергией. Последние повышают внутреннюю энергию торцевого слоя $U$, что эквивалентно повышению энергии когезии этого слоя. Повышение внутренней энергии или энергии когезии $E_{k n}$ можно оценить по следующему соотношению:

$$
U=E_{k n}=k \times n \times x \times \Delta U,
$$

где $k$ - количество участвующих в зацеплении полимерных молекул; $n-$ число связей $\mathrm{C}-\mathrm{C}$ в последовательности гош- и транс-изомеров; $x-$ средняя концентрация гош-изомеров; $\Delta U-$ энергия гош-изомера, равная $0.21 \mathrm{~kJ} / \mathrm{mol}$ [10].

Следует заметить, что торцевая поверхность наномостиков формируется в межламелярных прослойках, в которых могут встречаться сегменты молекул в различ- 


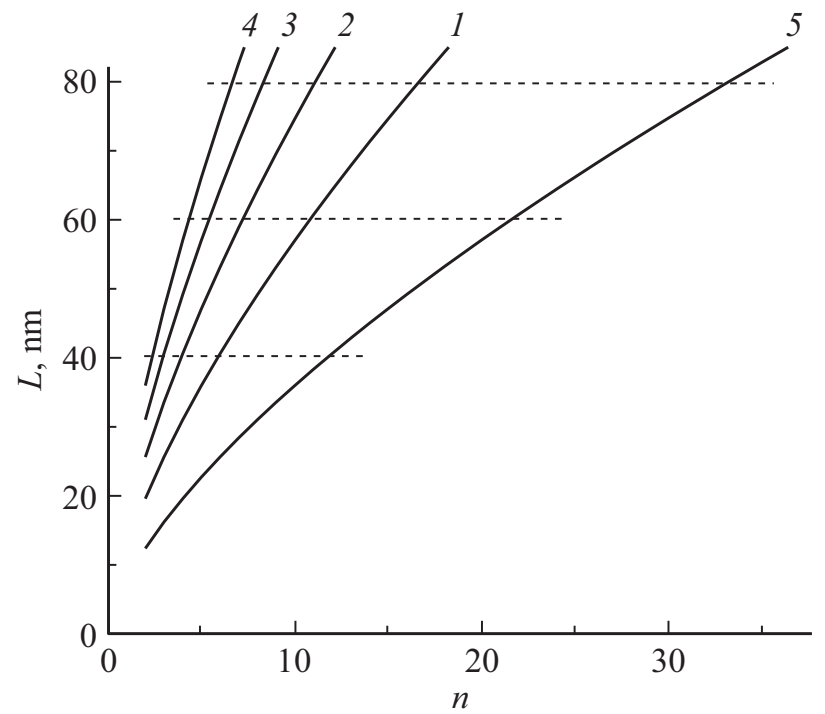

Рис. 4. Зависимости продольного размера $L$ зародыша ОФ от числа связей $\mathrm{C}-\mathrm{C}$.

ной конформации, задаваемой разным набором и последовательностью $t, g$ и $g^{*}$ изомеров, что обеспечивает изогнутую форму того или иного элемента структуры. В макрофибриллах ситуация иная - в межкристаллитных областях молекулы полимера находятся в ориентированном состоянии. Это состояние поддерживается определенным типом дефектов - „кинками“, в которых $t, g$ и $g^{*}$ изомеры располагаются в цепи полимера в строгой последовательности и концентрация $g$ изомеров равна концентрации $g^{*}$ изомеров.

Из соотношения (3) видно, что внутренняя энергия торцевого поверхностного слоя фибриллярного образования зависит от нескольких параметров. Комбинация этих параметров не должна приводить к значениям $U$, превосходящим энергию разрыва $\mathrm{C}-\mathrm{C}$-связи, и, соответственно, величину продольного размера фибриллярного образования больше $80 \mathrm{~nm}$. На рис. 4 представлены зависимости величины $L$ от $n$, полученные по соотношениям (2) и (3) при значении $x=0.5$, отвечающему состоянию полимерной цепи в точке зацепления, когда транс-изомеры чередуются с гош-изомерами ( $g$ или $\left.g^{*}\right)$. Кривые $1-4$ рассчитаны при $k=2,3,4$ и 5, то есть для двух, трех, четырех и пяти участвующих в зацеплении полимерных молекул, соответственно, при средней концентрации гош-изомеров $x=0.5$. Из рисунка видно, что увеличение числа зацеплений $(n)$ приводит к повышению избыточной энергии, при котором возможно образование наномостиков с различным $L$. Однако, число этих конфигураций ограничено величинами, кратными толщине одной ламели, то есть $L_{0}$.

Из рисунка видно, что при определенных сочетаниях $n$ и $k$ продольный размер наномостиков может достигать 2-х, 3-х или 4-х кратной толщины одиночных ламелей $(20 \mathrm{~nm})$. При этом элементарный объем фазового превращения $\omega$ в этих случаях один и тот же, меняется только $L$ наномостика. Для оценки числа $\mathrm{CH}_{2}$-групп, участвующих в образовании зародыша новой фазы, необходимо оценить объем одной $\mathrm{CH}_{2}$-группы $\left(\omega_{0}\right)$. Этот объем равен произведению площади сечения метиленовой цепи $(S)$ на проекцию длины последней на ось молекулы, то есть $\omega_{0}=h \times S$. Для расчета можно воспользоваться следующими данными: $S \sim 0.2 \mathrm{~nm}^{2}, h=0.125 \mathrm{~nm}[10]$, которые дают значение $\omega_{0} \sim 0.025 \mathrm{~nm}^{3}$. Сравнение $\omega_{0}$ и $\omega_{2}$ показывает, что в элементарном объеме превращения содержится $\sim 2200 \mathrm{CH}_{2}$-групп. При длине наномостика $\sim 80 \mathrm{~nm}$ в $\omega$ будет содержаться 3-4 проходных цепей.

В макрофибриллах сегменты макромолекул находятся в основном в конформации транс-зигзага, поэтому существование узлов зацепления молекул между собой в них менее вероятно, чем в неупорядоченных межламелярных прослойках. Однако, при достаточной концентрации „кинков“ в этих макрофибриллах внутренняя энергия распрямленных молекул может возрасти настолько, что этого окажется достаточно для формирования элементарного объема превращения, который формируется в наномостиках. Для проверки этого утверждения воспользуемся соотношениями (2) и (3) для одиночной цепи $(k=1)$ с чередованием изомеров $t-g-t-g^{*}-t(x=0.5)$. На рис. 4 (кривая 5) показан результат расчета функции $L(n)$ с использованием приведенных выше параметров. Видно, что наличие большого количества кинков и двойных перегибов может приводить к возникновению заметных внутренних напряжений и способствовать образованию моноклинной фазы. При этом участки цепей, в которых накапливается избыточная энергия, будут более протяженными, чем в наномостиках.

Проведенный в настоящей работе анализ тепловых эффектов, сопровождающий твердотельный фазовый переход в СВМПЭ из нестабильной МФ в термодинамически устойчивую ОФ, показал следующее: 1) в исходном реакторном порошке возможно существование МФ в виде наномостиков, которые не наблюдаются на растровых микрофотографиях. Эти наномостики соединяют между собой от 2-х до 4-х отдельных ламелей, 2) возможно существование МФ внутри макрофибрилл, наблюдаемых на микрофотографиях, 3) в компактированом СВМПЭ доля МФ резко возрастает, однако размер элементарного объема превращения МФ $\rightarrow$ ОФ указывает на мелкодисперсный характер распределения МФ внутри ламелей, что видно из соотношения элементарных объемов превращения (таблицу).

\section{Финансирование работы}

Работа выполнена при поддержке программ РФФИ 18-29-17023мк и 19-03-00789.

\section{Конфликт интересов}

Авторы заявляют об отсутствии конфликта интересов. 


\section{Список литературы}

[1] P. Smith, H.D. Chanzy, B.P. Rotzinger. Polym. Commun. 26, 258 (1985).

[2] P. Smith, H.D. Chanzy, B.P. Rotzinger. J. Mater. Sci. 22, 523 (1987).

[3] Yong Lak Joo, Huajun Zhou, Seung-Goo Lee, Hwan-Koo Lee, Jae Kyung Song. J. Appl. Polym. Sci. 98, 718 (2005).

[4] G. Forte, S. Rastogi, S. Ronca, H.J. Tjaden. The Netherlands patent WO 2012072780 A1 (2012).

[5] М.В. Байдакова, П.В. Дороватовский, Я.В. Зубавичус, Е.М. Иванькова, С.С. Иванчев, В.А. Марихин, Л.П. Мясникова, М.А. Яговкина. ФТТ 60, 1411( 2018).

[6] В.В. Аулов, М.А. Щербина, С.Н. Чвалун, С.В. Макаров, И.О. Кучкина, А.А. Пантюхин, Н.Ф. Бакеев, Ю.С. Павлов. BMC 46, 1005 (2004).

[7] Y.L. Joo, O.H. Han, H.-K. Lee, J.K. Song. Polymer 41, 1355 (2000).

[8] K. Illers. Eur. Polymer J. B 10, 911 (1974).

[9] V.A. Bershtein, V.M. Egorov. Differential Scanning Calorimetry of Polymers. Ellis, N-Y, Horwood (1994).

[10] Б. Вундерлих. Физика макромолекул. Плавление кристаллов. Мир, М. (1984). Т. 3. С. 64.

[11] P. Flory. J. Pol. Sci. B 49, 105 (1961).

[12] С.Я. Френкель. Энциклопедия полимеров. Сов. энциклопедия, М. (1974). Т. 2. С. 100.

[13] T. Yemny. R.L. McCullough. J. Pol. Sci. Pol. Phys. Ed. 11, 1385 (1973).

[14] М. Фишер. Природа критического состояния. Мир, М. (1968). $221 \mathrm{c}$

[15] Б.Н. Ролов, В.Э. Юркевич. Физика размытых фазовых переходов. Изд-во Рост. ун-та., Ростов (1983). 317 с.

[16] Г.А. Малыгин. Успехи физ. наук 171, 187 (2001).

[17] Г.А. Малыгин. ФТТ 43, 1911 (2001).

[18] В.М. Егоров, В.А. Марихин. ФТТ 60, 1441 (2018).

[19] В.М. Егоров, В.А. Марихин. ФТТ 58, 2269 (2016).

[20] D.W. VanKrevelen. Properties of polymers correlations with chemical structure. N.-Y. (1972). 480 p.

Редактор К.В. Емцев 